\title{
Ritodrine and Isoxsuprine in Management of Preterm Labor
}

\author{
Yogol NS, ${ }^{1}$ Shakya R, ${ }^{1}$ Thapa $\mathrm{P}^{1}$ \\ 'Department of Pharmacy, Kathmandu University, Dhulikhel, Kavre.
}

\begin{abstract}
Introduction: Preterm delivery is an important cause of perinatal morbidity and mortality. To decrease the medical and economical impact of preterm delivery, tocolytic agents are available among which isoxsuprine and ritodrine are widely used in Nepal. The study on efficacy of ritodrine and isosuprine has not been done yet in Nepelese women. So to observe on efficacy and safety of Ritodrine and Isoxsuprine, this study was conducted.
\end{abstract}

Methods: A prospective observational study was conducted with an aim to assess the efficacy and safety of Isoxsuprine $10 \mathrm{mg}$ orally eight hourly versus Ritodrine 10mg initially infused intravenously along with $5 \%$ dextrose at the rate of 10 drops per minute with an increase of 5-10 drops every 30 minutes for 24 hours and then given oral at 5-10 mg eight hourly, in patient with preterm labor requiring uterine tocolysis.

Results: This study found that Ritodrine is more effective and safer than Isoxsuprine in suppressing preterm labor. The failure rate of Isoxsuprine and Ritodrine were $22.22 \%$ and $6.5 \%$ respectively. The maternal side effects including cardiac side effects were significantly higher in Isoxsuprine. The cardiac side effects caused by Ritodrine can be controlled by adjusting the drip rate. Though Isoxsuprine seems more economical than Ritodrine, it is lesser cost effective to patients due to its higher failure rate, that results in added expenses in terms of increased morbidity and mortality.

Conclusions: Hence, Ritodrine is superior to Isoxsuprine as it is more efficacious in managing preterm labor and increasing fetal maturity. Also the adverse effects of Ritodrine are less than those of Isoxsuprine which result in better patient compliance and cost effectiveness.

Key Words: cost effectiveness, efficacy, preterm delivery, preterm labor, safety, tocolytic agents

\section{INTRODUCTION}

Preterm labor and the attendant complications are most common, costly and catastrophic complication of pregnancy. ${ }^{1}$ Preterm labor is defined as labor starting spontaneously after the gestation of 24 weeks to before
37 completed weeks of pregnancy. The majority of infants at this gestational period weigh less than $2500 \mathrm{gm}^{2}$

Ritodrine hydrochloride, a specific betamimetic tocolytic

\author{
Correspondence: \\ Ms. Nisha Shrestha Yogol \\ Kathmandu University \\ Dhulikhel, Kavre. \\ Email: nisha_shrestha@hotmail.com \\ Phone: $977-1-4486475$
}


agent, is associated with fewer maternal side effects. ${ }^{3,4}$ Isoxsuprine is a slight selective $\beta_{2}$-adrenoreceptor tocolytic agent, whose use has been superseded by more selective $\beta_{2}$-agonists. ${ }^{5,6}$ Studies have shown that Ritodrine is more efficacious in arresting preterm labor, delaying delivery and increasing fetal maturity than Isoxsuprine. ${ }^{6-8}$ Isoxsuprine is still used in developing countries due to its lower price. Though Ritodrine is affordable to the patient it is comparatively expensive. The higher failure rate and the related consequences of Isoxsuprine make treatment with Ritodrine more cost effective. The failure of preterm labor management results in preterm birth or infant mortality. Preterm birth accounts for $70 \%$ neonatal morbidity and increase expenses on neonatal care. ${ }^{9}$ Many studies have shown that Isoxsuprine has not only caused increased neonatal morbidity but also shows maternal and fetal side effects. ${ }^{7-10}$

\section{METHODS}

The prospective observational study was conducted at Gynecology and Obstetric ward of Dhulikhel Kathmandu University Teaching Hospital and Ante-neonatal Care Unit of Maternity Hospital, Thapathali from January 2007 to June 2007. This study was ethically approved by ethical review committee Kathmandu University, School of Medical Science.

With informed consent, a total of sixty-one pregnant women were included in the study. The inclusion criteria were: nulliparous or multiparous ladies in between a gestational age of 28-36 weeks, bishops score less than or equal to six and with symptoms and signs of preterm labor. The patients with cardiovascular disease or asthma or antepartum bleeding were excluded from the study. Bishops score was assessed to confirm whether patient is in active stage of labor or not. Since Maternity Hospital at Thapathali and Dhulikhel Hospital have their own protocol for treatment of preterm labor, the patients were assigned according to the treatment protocol of concerned hospitals. The subjects at Maternity Hospital, Thapathali $(n=30)$ were treated with Isoxsuprine $10 \mathrm{mg}$ every eight hours. The subjects at Dhulikhel Hospital $(n=31)$ were treated with intravenous infusion of Ritodrine $10 \mathrm{mg}$ with dextrose $5 \%$ at the rate of ten drops per minute $(50 \mathrm{mcg} / \mathrm{min})$ and the drip rate was increased by 5-10 drops every hour followed by oral administration of $5-10 \mathrm{mg}$ Ritodrine eight hourly depending on the severity of patient. Maternal pulse, blood pressure, fetal heart rate and uterine contraction were monitored prior to drug administration, then hourly for first six hours of initial dosing and then two hourly till the last dose. In case of the subject receiving intravenous infusion of Ritodrine, the drip rate was adjusted if the pulse rate was more than 100 per minute. When there was no control in labor pain despite tocolytic therapy, the subjects were subjected to elective cesarean section.

Baseline evaluation included height, weight, maternal age, socioeconomic status, literacy, gravidity, parity, gestational period, age at marriage, previous history of spontaneous abortion or preterm delivery, symptoms of urinary tract infection, history of smoking and alcohol consumption and underlying medical and obstetric disorders.

The efficacy was predicted with diminution of abdominal pain and backache with absence of vaginal discharge and uterine contraction. The safety of drugs was assessed with abnormal fetal and maternal heart rate and maternal side effects. The total cost of management was calculated.

Statistical analysis was done with SPSS version 11.5 data sheet. Pearson Chi Square test was used to compare efficacy, prolongation of pregnancy and adverse effects of drugs. Monitoring parameters pre-and post- use of drug were compared by using paired sample t-test. Mean and median were used as statistical tools to compare cost of treatments.

\section{RESULTS}

Among 31 patients treated with Ritodrine, the preterm labor management was successful and reached term delivery in 29 (93.5\%) patients whereas among the patients treated with Isoxsuprine, the treatment was successful only in 22 (73.3\%) patients out of 30 patients with $P$ value 0.033 (Figure 1,2). Among them $60 \%$ were primiparous (Table 1).

Table 1. Parity distribution of patients at preterm labor

\begin{tabular}{lll}
\hline Parity & Isoxsuprine* $^{*}$ & Rit o drine † \\
\hline Primiparous & $18(60 \%)$ & $19(61.3 \%)$ \\
Multiparous & $12(40 \%)$ & $12(38.7 \%)$ \\
\hline
\end{tabular}

${ }^{*} \mathrm{n}=30, \quad+\mathrm{n}=31$

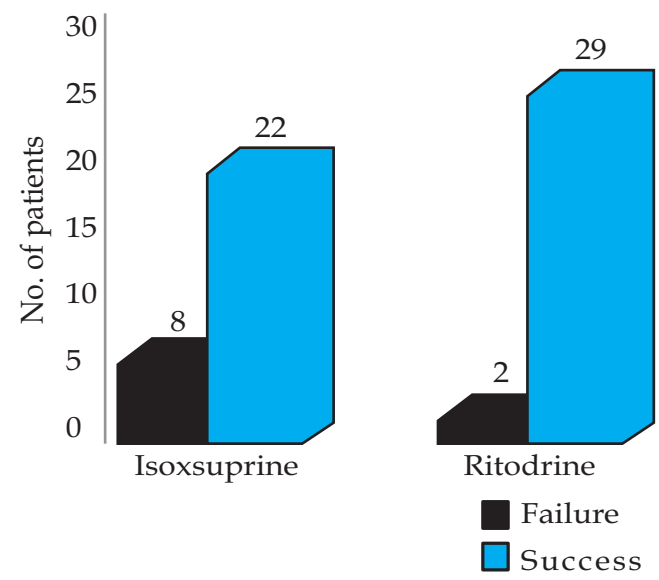

Figure 1. Effectiveness of tocolytic agents 


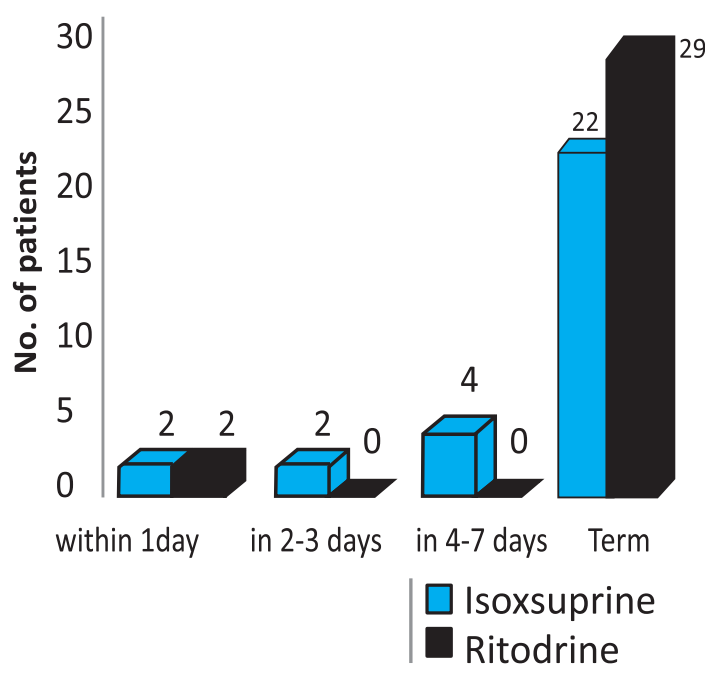

Figure 2. Prolongation of pregnancy

The study showed that more number of patients experienced adverse effects with Isoxsuprine than with Ritodrine. Incidences of nausea, vomiting and weakness with Isoxsuprine were found to be significantly higher than with Ritodrine (Table 2). Similarly the incidence of cardiac side effects such as tachycardia and/or hypotension was also significantly higher in Isoxsuprine group with $P$ value 0.033 (Table 3). Eight (26.7\%) out of 30 treated patients with Isoxsuprine experienced cardiac side effects (hypotension and/ or tachycardia) whereas only two $(6.5 \%)$ out of 31 patients treated with Ritodrine experienced same cardiac side effects.

Table 2. Maternal adverse effects

\begin{tabular}{llll}
\hline Adverse Effect & Isoxsuprine* & Ritodrine $\dagger$ & P-value \\
\hline Nausea & $12(40 \%)$ & $1(3.2 \%)$ & 0.0004 \\
Vomiting & $6(20 \%)$ & $1(3.2 \%)$ & 0.039 \\
Weakness & $5(16.7 \%)$ & - & 0.017 \\
Dry mouth & $1(3.3 \%)$ & - & 0.305 \\
Headache & $2(6.7 \%)$ & $2(6.5 \%)$ & 0.972 \\
Nervousness & $2(6.7 \%)$ & - & 0.143 \\
\hline
\end{tabular}

${ }^{*} \mathrm{n}=30, \quad+\mathrm{n}=31$

Table 3. Cardiac side effects

\begin{tabular}{llll}
\hline Cardiac side effects & Isoxsuprine* & Ritodrine $\dagger$ & P-value \\
\hline Present & $8(26.67 \%)$ & $2(6.45 \%)$ & 0.033 \\
Absent & $22(73.33 \%)$ & $29(93.55 \%)$ & \\
${ }^{*} \mathrm{n}=30,+\mathrm{n}=31$ & & &
\end{tabular}

The study showed no significant changes in pre-drug and post-drug monitoring parameters (maternal blood pressure, pulse and fetal heart rate) with either Isoxsuprine or Ritodrine. However, uterine contraction was significantly reduced after administration of drugs in both groups (Table 4).
Table 4. Changes in pre-drug and post drug monitoring parameters

\begin{tabular}{|c|c|c|c|c|}
\hline $\begin{array}{l}\text { Change in parameters } \\
\text { (Pre drug- Post drug) }\end{array}$ & Isoxsuprine* & P-value & Ritodrine $\dagger$ & P-value \\
\hline Systolic BP & $0.2 \pm 7.44$ & 0.884 & $3.16 \pm 10.99$ & 0.12 \\
\hline Diastolic BP & $0.7 \pm 8.04$ & 0.637 & $0.07 \pm 10.93$ & 0.974 \\
\hline Pulse/min & $1.66 \pm 6.50$ & 0.489 & $3.35 \pm 13.93$ & 0.19 \\
\hline No of Uterine Contraction & $0.4 \pm 0.68$ & 0.003 & $0.52 \pm 0.63$ & 0 \\
\hline $\mathrm{FHS} / \mathrm{min}$ & $0.3 \pm 4.07$ & 0.689 & $0.77 \pm 12.20$ & 0.726 \\
\hline
\end{tabular}

In this study, the duration of treatment with Isoxsuprine ranged from three to 21 days (median seven days) and that with Ritodrine ranged from two to 12 days (median six days). The average cost of treatment per patient with Isoxsuprine was $\$ 0.62(1 \$=N . R s .74)$ and that with Ritodrine was $\$ 5.0$ (including cost of intravenous set, intravenous canula and injection $5 \%$ Dextrose). The cost involved in Isoxsuprine treatment as compared to Ritodrine treatment was found to be in the ratio of $1: 8$.

\section{DISCUSSION}

The success rate of preterm labor management was significantly greater for Ritodrine compared to Isoxsuprine $(P=0.033)$. A similar study carried by Roy et al showed significant difference in effectiveness between two groups. Their study found that tocolysis was successful in $24(96 \%)$ and 21 (84\%) patients with Ritodrine and Isoxsuprine therapy respectively. ${ }^{7}$ The study carried out by Ikeda et al also showed that the efficacy of Ritodrine was greater than that of Isoxsuprine. ${ }^{8}$ Study carried out by Sirohiwal et al found that tocolysis with Ritodrine was successful in $76.7 \%$ patients and with Isoxsuprine it was successful in $63.3 \%$ patients. ${ }^{6}$ The results are consistent with the findings of this study.

In the present study, when duration of prolongation of pregnancy was compared, Ritodrine was found to be more effective. The study conducted by Sirohiwal et al found that there were $43 \%$ and $33 \%$ of term deliveries in-patient assigned for Ritodrine and Isoxsuprine respectively. ${ }^{6}$ Another study has also found more number of patients allocated to Ritodrine group reached term pregnancy. $^{7}$

The present study found a higher incidence of occurrence of adverse effects with Isoxsuprine than with Ritodrine, so Ritodrine can be considered as relatively safer to the patient. The study carried out by Roy et al also found that patients in Ritodrine group experienced fewer side effects compared to Isoxsuprine treated patients. ${ }^{7}$ The study carried out by lkeda et al showed that adverse effects like hypotension and tachycardia were lesser with Ritodrine than with Isoxsuprine. ${ }^{8}$ The study conducted by Barden et al showed that Ritodrine seems to be relatively safer and side effects are usually tolerable 
compared to Isoxsuprine. ${ }^{11}$

There are no significant changes in pre- drug and post drug monitoring parameters with either Isoxsuprine or Ritodrine. However, uterine contraction was significantly reduced after administration of drugs in both Ritodrine and Isoxsuprine group which indicates uterine relaxant effect of both drugs. Despite this, the rate of diminution of number of uterine contractions was found to be higher with Ritodrine than with Isoxsuprine which indicates that Ritodrine has more rapid onset of action than Isoxsuprine. This difference in onset of action could be due to the difference in route of administration as Ritodrine was initially administered intravenous while Isoxsuprine was given orally. However, the results of this study suggest that Ritodrine more effectively suppresses uterine motility and also has lesser effects on maternal cardiovascular system.

The direct cost of treatment with Ritodrine is higher than that with Isoxsuprine; but it is still affordable to the patient. The study carried out by Roy et al showed the cost involved in Isoxsuprine treatment as compared to Ritodrine treatment is in the ratio of $1: 3 .^{7}$ In that study, Isoxsuprine was used in injectable form as starting dose followed by oral tablets after two to three days. In present study only oral tablets of Isoxsuprine was used, which could have resulted in the difference in cost of treatment between the two studies.

The study showed Ritodrine has higher success rate than Isoxsuprine in preventing preterm deliveries $193.5 \%$ vs $73.0 \%)$. It is well accepted that the consequences of failure of tocolysis are neonatal morbidity and mortality, which cost more than the direct cost of treatment of preterm labor either with Ritodrine or with Isoxsuprine.
Again the incidences of overall adverse effects including maternal cardiac side effects such as tachycardia and hypotension were also significantly higher in Isoxsuprine $(26.7 \%)$ than in Ritodrine $(6.5 \%)$. Several studies have shown that Isoxsuprine has limited therapeutic value with unpleasant side effects and efficacy. ${ }^{7,8,10,12}$ Hence, Ritodrine seems to be a much safer tocolytic agent than Isoxsuprine. Although the direct cost of treatment with Ritodrine is higher than that with Isoxsuprine, it is still bearable to the patient. Hence, it could be the more preferable betamimetic agent used as tocolytic agent rather than Isoxsuprine.

Since the sample size of this study was small and the study was conducted in only two hospitals of Nepal for short duration, the study cannot be delineated to whole population. Randomized control trials would have produced more reliable results.

\section{CONCLUSIONS}

The study found that Ritodrine was significantly successful in arresting preterm labor than Isoxsuprine. The study also found that Ritodrine was comparatively safer than Isoxsuprine in terms of occurrence of cardiac side effects as well as extra cardiac maternal side effects. Although cost of Ritodrine was higher than cost of Isoxsuprine, it was justifiable in long term in view of increased economic burden of tocolytic failure and increased adverse effects.

\section{ACKNOWLEDGEMENTS}

Staff of Obstetrics and Gynecology Department of Dhulikhel Hospital, Maternity Hospital Thapathali and staffs of Pharmacy Department, Kathmandu University.

\section{REFERENCES}

1. Morrison JC. Preterm Labor: Prediction and Treatment. Obstetrics and Gynecology Clinics of North America. 2005;32(3):369-81.

2. Gabby SG, Niebyl JP, Simpson JL. Preterm birth. Obstetrics, Normal \& Problem Pregnancies, 4th ed. Philadelphia: Churchill Livingstone; 2002. p. 787-91.

3. Goldenberg RL \& Rouse DJ. Prevention of preterm birth. N Engl J Med 1998;339:313-20.

4. Papatsonis DNM, Van Geijn HP, Ader HJ, Lange FM, Bleker OP, Dekker GA. Nifedipine and Ritodrine in the management of preterm labor: a randomized multicenter trial. Obstet Gynecol. 1997;90:230-4.

5. Gennaro AR, Chase GD, Marderosian AD, Hanson GR, Hussar DA. Remington: The Science \& Practice of Pharmacy. 19th ed. USA: Mack Publishing Company; 1993. p. 992,998.

6. Sirohiwal D, Sachan A, Bano A, Gulati N. Evaluation of ritodrine for tocolysis in preterm labor. J Obs Gyn. 2001;51:66-7.
7. Roy V, Prasad GS, Latha K. Tocolysis with Ritodrine: A Comparative Study in Preterm Labor. Pakistan J Medical Sciences. 2006;22:1.

8. Ikeda S, Tamaoki H, Akahane M, Nebashi Y. Effect of ritodrine hydrochloride, a beta 2- adrenoreceptor stimulant, on uterine motilities in late pregnancy. Jpn J Pharmacol. 1984; 35(3):31926.

9. Newton ER. Preterm labor, preterm premature rupture of membranes and chorioamnionitis. Clin Perinatol. 2005 Sep; 32(3):571-600.

10. Kazzi NJ Gross TL Kazzi GM Williams TG. Neonatal complication following in utero exposure to intravenous ritodrine. Acta Obstet Gynecol Scand. 1987;66(1):65-9.

11. Merkatz IR, Peter JB, Barden TP. Ritodrine hydrochloride: a betamimetic agent for use in preterm labor. II. Evidence of efficacy. Obstet Gynecol. 1980;56(1):7-12.

12. King JF, Grant A, Keirse MJ, Chalmers I. Betamimetics in preterm labor. An overview of randomized controlled trials. Br J Obstet Gynaecol. 1998;95:211-22.

\section{\begin{tabular}{l|l|l|l|l|l|l|l|} 
JNMA & VaL 48 & Na. 4 & ISSUE & 176 & DCT-DEC, 2009
\end{tabular}}

